\title{
A Causal Role for the Extrastriate Body Area in Detecting People in Real-World Scenes
}

\author{
Martijn G. van Koningsbruggen, ${ }^{1,2,3}$ Marius V. Peelen, ${ }^{2}$ and Paul E. Downing ${ }^{1}$ \\ ${ }^{1}$ School of Psychology, Bangor University, Bangor Gwynedd, United Kingdom LL57 1UT, and ${ }^{2}$ Center for Mind/Brain Sciences and ${ }^{3}$ Department of \\ Cognitive Sciences, University of Trento, 38068 Rovereto, Italy
}

People are extremely efficient at detecting relevant objects in complex natural scenes. In three experiments, we used functional magnetic resonance imaging-guided transcranial magnetic stimulation (TMS) to investigate the role of the extrastriate body area (EBA) in the detection of people in scenes. In Experiment 1, participants reported, in different blocks, whether people or cars were present in a briefly presented scene. Detection (D-prime) of people, but not of cars, was impaired after TMS over right EBA (rEBA; five pulses at - 200, - 100, $0,100,200 \mathrm{~ms})$ compared with sham stimulation. In Experiment 2, we applied TMS either before $(-200,-100 \mathrm{~ms})$ or after $(+100,+200)$ the scene onset. Poststimulus EBA stimulation impaired people detection relative to prestimulus EBA stimulation, while timing had no effect during sham stimulation. In Experiment 3, we examined anatomical specificity by comparing TMS over EBA with TMS over scene-selective transverse occipital sulcus (TOS). Two scenes were presented side by side, and response times to detect which scene contained people (or cars) were measured. For people detection, but not for car detection, response times during EBA stimulation were significantly slower than during TOS stimulation. Furthermore, rEBA stimulation led to an equivalent slowing of response times to left and right lateralized targets. These findings are the first to demonstrate the causal involvement of a category-selective human brain region in detecting its preferred stimulus category in natural scenes. They shed light on the nature of such regions, and help us understand how we efficiently extract socially relevant information from a complex input.

\section{Introduction}

An important capacity of human vision is the ability to rapidly extract relevant information from complex natural scenes. Human observers can accurately make judgments about briefly presented scenes, such as the semantic category of the depicted location or the presence of an abstractly specified target (Intraub, 1981; Thorpe et al., 1996). More recently, neuroimaging approaches have begun to show how the process of searching and categorizing scenes arises from a coordination of activity in posterior and anterior brain regions (Peelen and Kastner, 2011). However, the causal role of specific brain regions in parsing natural scenes remains poorly understood.

As a social species, one of the key elements we must extract from natural scenes is information about the conspecifics around us. Perhaps the most basic question that a "social brain" must ask of the surrounding environment is as follows: "is there someone else there?" Accordingly, the bodies and faces of other individuals are salient, tending to capture the gaze and attention of observers (Downing et al., 2004; Ro et al., 2007; Bindemann et al., 2010; Stein et al., 2012). The importance of perceiving human bodies

Received June 15, 2012; revised Feb. 20, 2013; accepted March 11, 2013.

Author contributions: M.G.V.K., M.V.P., and P.E.D. designed research;M.G.V.K. performed research; M.G.V.K. and P.E.D. analyzed data; M.G.V.K., M.V.P., and P.E.D. wrote the paper.

This research was supported by the Leverhulme Trust. We thank Robert Rafal and Nick Oosterhof for helpful discussions and Tegan Penton for help with data collection.

Correspondence should be addressed to Martijn van Koningsbruggen, Center for Mind/Brain Sciences (CIMeC), University of Trento, 38068 Rovereto (TN), Italy. E-mail: mg.vankoningsbruggen@unitn.it.

DOI:10.1523/JNEUROSCI.2853-12.2013

Copyright $\odot 2013$ the authors $\quad 0270-6474 / 13 / 337003-08 \$ 15.00 / 0$ and faces appears to be mirrored in the organization of high-level visual regions in extrastriate cortex. In functional magnetic resonance imaging (fMRI) studies of humans and macaques, several focal regions respond strongly and selectively to images of these stimuli, relative to other objects (Kanwisher and Yovel, 2006; Peelen and Downing, 2007; Tsao and Livingstone, 2008).

Here we focus on one such region, the extrastriate body area (EBA; Downing et al., 2001), found in the posterior lateral occipitotemporal cortex, most strongly in the right hemisphere. EBA likely plays a role in the perception of the shape and posture of human bodies and body parts (Downing and Peelen, 2011), and has been proposed to be involved in body detection rather than identification (Chan et al., 2004; Hodzic et al., 2009). To date, efforts to demonstrate a causal role for EBA in perception have mainly applied transcranial magnetic stimulation (TMS). In several studies, TMS over EBA, relative to control sites, shows specific impairments in accuracy and response time for discrimination tasks on body stimuli, relative to other categories (Urgesi et al., 2004, 2007a,b; Pitcher et al., 2009; Calvo-Merino et al., 2010). These studies typically used a delayed match to sample discrimination task, with stimulation applied in the interval or at test. These tasks required discrimination of fine differences in body posture or shape on stimuli that were presented in isolation on a homogenous background. Hence they do not address the potential role of EBA in the detection of people in natural scenes.

In the present study, we consistently find that stimulation of EBA interferes selectively with person detection in natural scenes. Furthermore, processing immediately after, as opposed to immediately before, scene presentation is critical for detection; inter- 
ference with right hemisphere EBA impedes detection equally in both hemifields. This study is the first to demonstrate and characterize the causal involvement of a category-specific brain region in the detection of preferred stimuli in natural scenes.

\section{Materials and Methods}

Participants. Right-handed participants were recruited from the Bangor student community. In an initial, separate session, the procedure and possible risks were explained to the participants. Next, written informed consent was obtained from each participant, and participants completed a safety screening questionnaire for TMS (Keel et al., 2001). Participants were familiarized with the sensation of repetitive TMS (rTMS) over the temporal lobe. Only participants who fulfilled all safety criteria, tolerated rTMS over the temporal lobe region, and whose performance was above D-prime $=1$ on a brief version of the main experiment, were allowed to participate. For three potential participants, task performance was inadequate, and a further two found the TMS too uncomfortable and so elected not to participate. Ethics approval was obtained from the School of Psychology at Bangor University, UK. Participants received $\mathfrak{E} 10 / \mathrm{h}$ for their participation.

Imaging methods for localization. Before the TMS experiment(s), each participant was scanned on a series of blocked-design localizer scans to identify functional regions of interest with respect to individual brain anatomy. Each scan consisted of 2116 s blocks in which blocks 1, 6, 11, 16 , and 21 were fixation-only baseline epochs. The stimuli consisted of blocks of images of human bodies (without heads), unfamiliar faces, outdoor scenes, and chairs. Each condition was presented in four $16 \mathrm{~s}$ blocks in each scan. In each block, 20 images (selected randomly from a full set of 40) from one category were presented (300 ms on/500 ms off). Twice during each stimulus block, the same image was presented two times in succession. Participants were instructed to detect these immediate repetitions and report them with a button press (1-back task). Each participant was scanned on at least two runs of this localizer. Two different versions were tested that were identical except for the order of blocks. Within each scan, the assignment of blocks to conditions was also counterbalanced so that the mean serial position in the scan of each condition was matched and centered.

The data were acquired using a $3 \mathrm{~T}$ Philips MRI scanner with an 8-channel SENSE phased-array head coil. For functional imaging, a single shot echo planar imaging sequence was used $\left(\mathrm{T} 2^{*}\right.$-weighted, gradient echo sequence; echo time, $35 \mathrm{~ms}$; flip angle, $90^{\circ}$ ). The scanning parameters were as follows: repetition time $2000 \mathrm{~ms} ; 35$ off-axial slices; voxel dimensions $3 \times 3 \mathrm{~mm} ; 3 \mathrm{~mm}$ slice thickness, SENSE factor 2, phase encoding direction anterior-posterior. The slices were positioned to include the entire cortex. Seven dummy volumes were acquired before each functional scan to reduce the effects of T1 saturation. Parameters for T1-weighted anatomical scans were as follows: 175 sagittally oriented slices; $1 \mathrm{~mm}$ isotropic voxels; $\mathrm{TR}=8.4 \mathrm{~ms}$, $\mathrm{TE}=3.8 \mathrm{~ms}$; flip angle $=8^{\circ}$.

Preprocessing and statistical analyses of the MRI data were performed using BrainVoyager QX (Brain Innovation). Functional data were motion corrected, and low-frequency drifts removed with a temporal highpass filter $(0.006 \mathrm{~Hz})$. Spatial smoothing was applied $(4 \mathrm{~mm}$ full-width at half-maximum). Functional data were automatically coregistered with the anatomical T1 scans. TMS coil positioning was based on fMRI data registered to $\mathrm{T} 1$ images in native space.

For each participant, a general linear model was created. One boxcar predictor, convolved with a two-gamma hemodynamic response function to model the hemodynamic response, modeled each condition of interest. Regressors of no interest were also included to account for differences in the mean MR signal across scans. Regressors were fitted to the MR time series in each voxel and the resulting $\beta$ parameter estimates were used to estimate the magnitude of response to each experimental condition.

In each participant, right hemisphere EBA was defined by contrasting the response to human bodies with that to the remaining three conditions. The right hemisphere EBA was selected as this region is normally found in nearly every individual, and has been successfully stimulated in previous TMS studies. In the present study we were able to identify a right
EBA region of interest in all participants. The mean Talairach and Tournoux (1988) coordinates (X, $Y, Z$, with SEs) for the targeted region were as follows: Experiment 1: 48 (1.0), -64 (2.6), -4 (1.2); Experiment 2: 44 (1.4), -65 (1.7), -3 (1.9); Experiment 3: 47 (1.1), -69 (1.6), 0 (2.1). In Experiment 3, a scene-selective region of transverse occipital sulcus (TOS; Epstein et al., 2005) was also defined, by contrasting the response to scenes with that to images of chairs. Peak voxels were selected within anatomical regions referring to previous studies (Epstein et al., 2005; Kontaris et al., 2009) and used to position the TMS coil (mean coordinates $=33(1.1),-77(2.0), 14(1.8))$.

General TMS methods. A MagStim Super Rapid Stimulator (Magstim) with a $70 \mathrm{~mm}$ figure-eight coil was used for the TMS. The hand area of motor cortex was first localized in the right hemisphere. We used the right hemisphere motor cortex/left hand for threshold determination in order that the same hemisphere would be used to set intensity levels and to perform the primary experiments. The motor threshold (MT) then was determined by finding the minimum amount of TMS intensity that was required to elicit a clearly visible hand twitch in the relaxed left, nondominant, hand in at least 5 of 10 attempts. Stimulation was set to $120 \%$ of the MT.

At the start of each block, the exact TMS location was determined by using Brainsight (Rogue Research) neuronavigation software, and was marked on a tight-fitting swimming cap. Sham TMS was given over the rEBA by tilting the coil in a $90^{\circ}$ angle from the skull. For TMS over rEBA or TOS, the handle of the coil was oriented upward; the exact orientation was adjusted individually to minimize muscle stimulation.

Stimuli. Natural scene pictures were selected from an on-line database (Russell et al., 2008) and were a subset of those used in previous fMRI studies (Peelen et al., 2009; Peelen and Kastner, 2011). Pictures could contain one or more people (but no cars), one or more cars (but no people), or no cars and no people. The pictures were primarily photographs of city streets. The position, viewpoint, and size of the people and cars in the pictures were highly variable, mimicking real-world viewing conditions (Fig. 1). Perceptual masks were colored pictures of a mixture of white noise at different spatial frequencies on which a naturalistic texture was superimposed (Walther et al. 2009). Within a TMS session each of the presented pictures was unique. All pictures were full-color photographs reduced to 480 (vertical) $\times 640$ (horizontal) pixels.

Experiment 1 procedure. To ensure that EBA could effectively be stimulated with TMS, participants were selected for whom TMS over rEBA caused impaired body discrimination performance in several different pilot experiments. These informal pilot experiments, adopted from previous studies (Urgesi et al., 2004; Pitcher et al., 2009), with identical stimuli and timings, but with only sham or TMS over rEBA, required participants to discriminate the shape of isolated body part stimuli using a delayed match-to-sample paradigm. We either used identical TMS stimulation $(N=22)$, or used off-line theta TMS instead $(N=4)$. Of these, $14 / 26$ (54\%) showed lower mean body discrimination performance after rEBA TMS, relative to sham TMS. These participants were asked to participate in Experiment 1; 12 of them (2 male; mean age 24 years, $\mathrm{SE}=1.3$ ) agreed to do so. By using this selection procedure, we ensured that TMS was likely to be effective in disrupting rEBA in our sample, excluding participants for whom TMS was unable to effectively target rEBA due to, for example, large skull-EBA distance, ineffective coil orientation, and/or TMS intensity that was too low. It should be noted, however, that our selection procedure potentially limits the generalizability of results, as our results only apply to those individuals who previously showed an effect of rEBA TMS on body discrimination (Urgesi et al., 2004, 2007a,b; cf. Pitcher et al., 2009).

Participants were tested in two sessions. The first session was used to titrate presentation times and TMS was not applied. In the second session, presentation time was fixed, and TMS (or sham TMS) was applied. Data from one participant were dropped due to extremely poor overall performance on the task (all D-primes $<1$ ); including this participant's data in the analysis did not change the overall pattern of results.

Participants were seated in front of a CRT computer screen $(1024 \times$ 768 pixel dimensions, $85 \mathrm{~Hz}$ refresh rate); a chin rest held viewing distance constant at $57 \mathrm{~cm}$. Each trial began with the presentation of an outline circular fixation point at the center of the screen, which indicated 
People
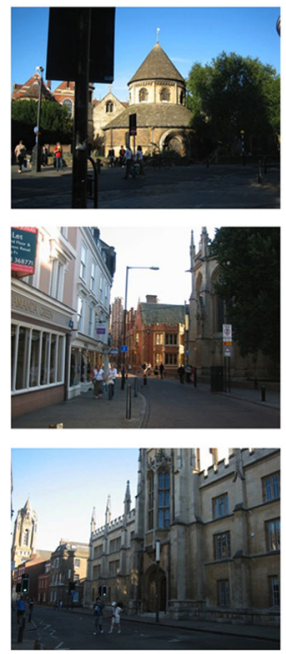

Cars
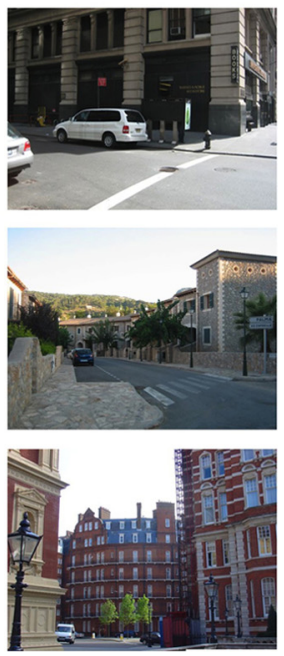

Neither
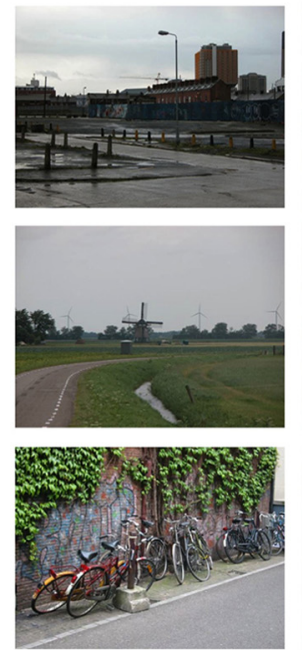

Figure 1. Sample real-world scenes used in Experiments 1-3. Columns, from left to right: scenes with people, with cars, with neither, and masks.

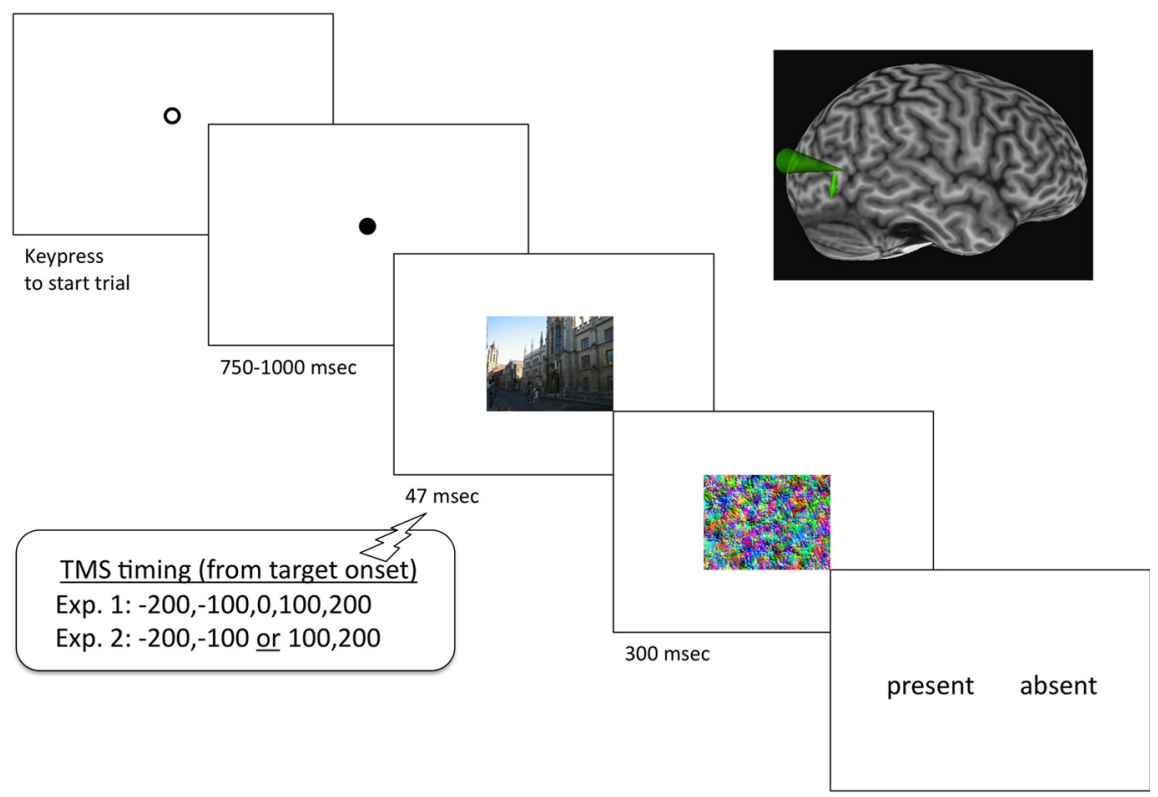

Figure 2. Diagram of experimental protocol from Experiments 1 and 2. Either TMS was delivered to right hemisphere EBA (functionally localized in each individual), or sham stimulation was applied. Figure elements are not to scale. Inset figure illustrates typical anatomical location of EBA in a screen capture from Brainsight stereotactic localization software. The small arrow reflects the estimated trajectory of the TMS coil.

that the trial was ready to begin (Fig. 2). When ready, participants initiated the trial with a keypress, at which point the outline fixation point was filled in (black). To reduce participants' ability to anticipate the stimulus, there was then a variable delay interval of 750-1000 ms during which only the fixation point was visible. Next, a single, full-color natural scene was presented centered on fixation and spanning $10^{\circ}$ width and $8^{\circ}$ height. In the first session, duration of this stimulus was varied in different blocks among 35, 47, and $59 \mathrm{~ms}$. Based on results from the first session, scene duration was set at $47 \mathrm{~ms}$ (four screen refreshes) for the second session. At this duration most participants performed above chance but below ceiling. On each trial in the second session, TMS pulses were delivered at -200 , $-100,0,+100$, and +200 ms relative to the onset of the scene image.

The scene was replaced by one of the mask images, selected randomly on each trial. The mask was presented for $300 \mathrm{~ms}$. Subsequently the words "present" and "absent" were presented to either side of the fixation
Masks
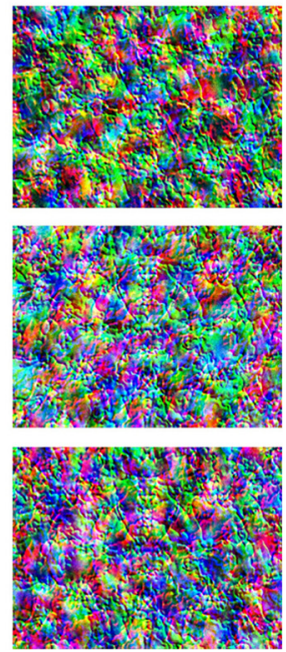

point, spatially compatible with the relevant response keys. Participants were asked to decide whether the target item for that block (person or car) was present; accuracy (not speed) was emphasized. An intertrial interval of $4 \mathrm{~s}$ was then enforced before participants could begin the next trial.

In both sessions, half of the participants started with the car task (for two blocks) and half with the people task. For the first session, 40 trials were tested for each of the three target durations in separate blocks. For the second session, stimulation type (rEBA or sham) and task (detect people or cars) were varied across four blocks of 108 trials each. Orthogonally, half of the participants were tested in the order \{sham, rEBA, sham, rEBA $\}$ or $\{\mathrm{rEBA}$, sham, rEBA, sham\}. A 2 min break was enforced every 36 trials. The coil was changed during every break to prevent overheating, and the stimulation location was checked.

Experiment 2 procedure. The participants in Experiment 2 had not taken part in Experiment 1 . They were selected from a group of 23 participants who participated in another TMS experiment (not reported here) that included, in addition to other conditions, conditions in which TMS was applied over EBA during person and car detection tasks. As in Experiment 1, TMS pulses were applied both before and after the onset of the scene image $(-200$, $-100,0,+100,+200 \mathrm{~ms}$ ), and $18 / 23$ participants $(78 \%)$ showed stronger effects of TMS on person detection than on car detection. The 9 participants in Experiment 2 (three male, mean age $=23, \mathrm{SE}=1.33$ ) were selected from these 18 participants.

The present study tested if decreased performance on the person-detection task following TMS over EBA was primarily the result of TMS interfering with the preparatory interval, the postprocessing interval, or both. Each participant was tested over 2 days. The first day consisted of a replication of Experiment 1 (Experiment 2a). On the second day (Experiment $2 \mathrm{~b}$ ), participants were tested in four blocks (90 trials each) of the person-detection task (the car task was not tested). Block types were determined from the factorial combination of \{pretarget or post-target stimulation $\} \times$ \{sham or rEBA stimulation site\}. In pretarget blocks, two TMS pulses were delivered, at -200 and -100 ms relative to the onset of the scene. In post-target blocks, two pulses were delivered at +100 and $+200 \mathrm{~ms}$. The order of blocks was counterbalanced across participants. In other respects the procedures were identical to Experiment 1 (Fig. 2).

Experiment 3 procedure. In Experiment $3(N=16$; nine new unselected participants (four male) and seven randomly selected from Experiments 1 or 2 (five male), mean age $=20, \mathrm{SE}=0.4$ ) we tested performance on a localization task (Fig. 3). On each trial, two natural scenes were presented, in gray scale, to the left and right of the fixation point. The distance between the two images, center to center, was $18^{\circ}$ visual angle. For the person task, one of the two scenes had a person or people in it, and the other did not; likewise for the car task. The target item appeared on either the left or the right with exactly $50 \%$ probability across each block, with the constraint that it never appeared on the same side $>5$ trials in a row. The two tasks were completed in two separate sessions ( $\sim 1$ week apart), counterbalanced for order across participants. 
The two scenes were presented for $94 \mathrm{~ms}$ (eight screen refreshes), after which they were each replaced with masks, which remained present until the participant responded. On each trial, participants were asked to report with a keypress which of the two scenes contained either people or cars, depending on the session. Participants were instructed to make speeded responses. Reaction time was measured relative to scene onset. Three blocks of 40 trials were tested in each session. During each block, stimulation was delivered at either right hemisphere EBA or right hemisphere TOS, or sham stimulation was applied. Five TMS pulses were given, time locked to the onset of the target scenes (e.g., $-200,-100,0,+100,+200 \mathrm{~ms}$ ).

\section{Results}

\section{Experiment 1}

The D-prime results were submitted to a within-participants ANOVA with Task (car, people) and TMS Site (EBA, sham) as factors (Fig. 4). There was a significant main effect of TMS Site, $F_{(1,10)}=21.3, p<$ $0.005, \eta_{p}{ }^{2}=0.68$, qualified by a significant interaction between both factors, $F_{(1,10)}=8.4, p<0.05, \eta_{p}{ }^{2}=0.46$. There was no significant main effect of Task, $F_{(1,10)}=1.1, p=0.32, \eta_{p}{ }^{2}=0.10$. Simple effects tests for each task revealed significantly lower D-prime on the people task for EBA stimulation $(1.63(\mathrm{SE}=0.18))$ compared with sham stimulation $(2.32(\mathrm{SE}=0.15)), t_{(10)}=5.5, p<0.001$. In contrast, there was no significant difference on the car task $(\mathrm{rEBA}=1.78(\mathrm{SE}=$ $0.09)$; sham $=1.91(\mathrm{SE}=0.15)), t_{(10)}=0.97$.

The signal detection framework allows us to separate D-prime (sensitivity to detect the presence or absence of a specified target in a natural scene) from criterion (level of evidence at which the observer is willing to report that a target is present). Criterion measures $(\mathrm{C}=-0.5 \times(\mathrm{zHit}+\mathrm{zFA}))$ were submitted to a within-participants ANOVA with Task (car, people) and TMS Site (EBA, sham) as factors. There were no significant main effects, all $F<1$. However, there was a significant interaction, $F_{(1,10)}=10.1, p<0.01, \eta_{p}{ }^{2}=0.50$. Simple effects tests for each task revealed significantly higher criterion on the people task for EBA stimulation $(0.44(\mathrm{SE}=0.13))$ compared with sham stimulation $(0.39(\mathrm{SE}=0.13)), t_{(10)}=2.82, p<0.05$. In contrast, there was a significantly lower criterion on the car task for EBA stimulation $(0.19(\mathrm{SE}=0.14))$ relative to sham $(0.39(\mathrm{SE}=0.15))$, $t_{(10)}=2.30, p<0.05$. Thus EBA stimulation made participants more conservative in the people task, and more liberal in the car task, relative to sham stimulation.

Reaction times were submitted to a within-participants ANOVA with Task (car, people), TMS Site (EBA, sham), and Trial Type (Hit, CR) as factors. There was a significant main effect of Trial Type, $F_{(1,10)}=31.2$, TMS Site, $p<0.005, \eta_{p}{ }^{2}=0.76$, caused by slower response times during CR trials (613 ms) compared with Hit trials (562 ms). In addition, there was a significant interaction between Task $\times$ TMS, $F_{(1,10)}=6.2, p<$ $0.05, \eta_{p}{ }^{2}=0.38$. Follow-up paired sample $t$ tests did not reveal any significant reaction time effect for the People task (Sham $(580 \mathrm{~ms}(\mathrm{SE}=42))$ versus $\mathrm{rEBA}(596 \mathrm{~ms}(\mathrm{SE}=36)), t<1)$, nor for the Car task (Sham $(599 \mathrm{~ms}(\mathrm{SE}=38))$ versus rEBA $(575$ $\left.\mathrm{ms}(\mathrm{SE}=40)), t_{(10)}=1.5, p=0.17\right)$. No other main effects or interactions were significant.

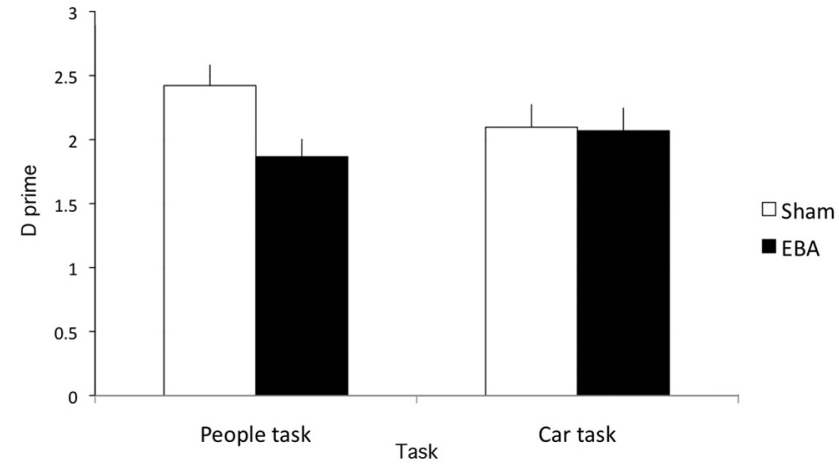

Figure 4. Results of Experiment 1. D-prime for detection of people (but not cars) in natural scenes is reduced by TMS over rEBA, relative to sham stimulation. This pattern was also replicated in a separate group of participants (see Results). Error bars in this and all following data figures indicate SEM.

\section{Experiment 2a}

D-prime results were submitted to a within-participants ANOVA with Task (car, people) and TMS Site (EBA, sham) as factors. There was a significant main effect of TMS Site, $F_{(1,8)}=8.5, p<$ $0.05, \eta_{p}{ }^{2}=0.51$, qualified by a significant interaction, $F_{(1,8)}=$ 14.6, $p<0.01, \eta_{p}{ }^{2}=0.65$. Simple effects tests for each task revealed significantly lower D-prime on the people task for EBA stimulation $(1.87(\mathrm{SE}=0.14))$ compared with sham stimulation $(2.42(\mathrm{SE}=0.16)), t_{(8)}=4.6, p<0.005$. In contrast, there was no significant difference on the car task $(\mathrm{rEBA}=2.07(\mathrm{SE}=0.18)$; sham $=2.10(\mathrm{SE}=0.18)), t_{(8)}=0.9$. Criterion results were submitted to a within-participants ANOVA with Task (car, people) and TMS Site (EBA, sham) as factors. There were no significant effects (all $F<1.1$ ).

\section{Experiment $2 b$}

D-prime results were submitted to a within-participants ANOVA with Timing (pre, post) and TMS Site (EBA, sham) as factors (Fig. 5). Neither main effect reached significance, $F_{(1,8)}=2.65$, 


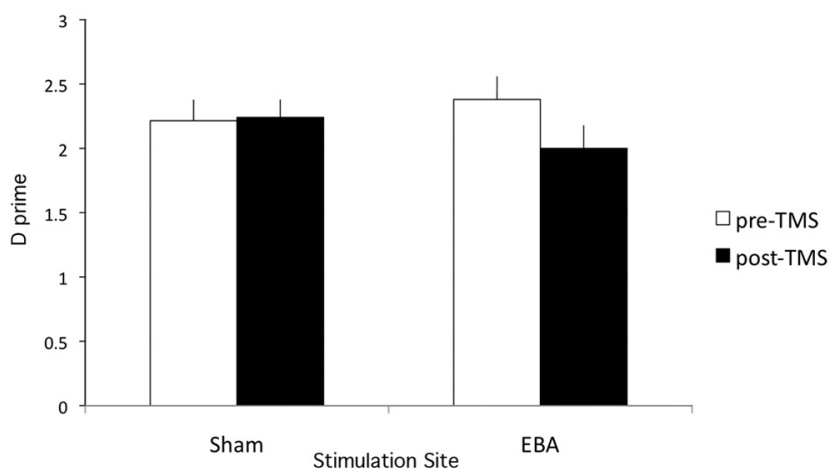

Figure 5. Results of Experiment 2b. Dual-pulse TMS over EBA immediately after $(+100$, $+200 \mathrm{~ms}$ ) stimulus presentation reduces D-prime for person detection, relative to TMS before $(-200,-100 \mathrm{~ms})$ presentation. No difference between time intervals was found for sham stimulation.

$\eta_{p}{ }^{2}=0.25$ and $F_{(1,8)}=0.12, \eta_{p}{ }^{2}=0.01$, respectively. Importantly, the interaction was significant, $F_{(1,8)}=5.6, p<0.05$, $\eta_{p}{ }^{2}=0.41$. For sham stimulation, there was no significant effect of timing (pre-TMS $=2.21(\mathrm{SE}=0.14)$; post-TMS $=2.24(\mathrm{SE}=$ $0.17)), t_{(8)}<1$. In contrast, for EBA stimulation, poststimulus TMS $(2.00(\mathrm{SE}=0.12))$ produced significantly worse performance than prestimulus TMS $(2.38(\mathrm{SE}=0.13)), t_{(8)}=2.9, p<$ 0.05 . Criterion results were submitted to a within-participants ANOVA with Timing (pre, post) and TMS Site (EBA, sham) as factors. Neither main effect reached significance, both $F<1$. The interaction of these factors was significant, $F_{(1,8)}=6.09, p<0.05$, $\eta_{p}{ }^{2}=0.43$. However, simple effects tests for each TMS condition separately revealed no significant differences (Pre-Sham $(0.26$ $(\mathrm{SE}=0.11))$ versus Post-Sham $(0.47(\mathrm{SE}=0.15)), t_{(8)}=1.77$, $p=0.11$; Pre-TMS $(0.43(\mathrm{SE}=0.18)$ versus Post-TMS $(0.29$ $\left.(\mathrm{SE}=0.18)), t_{(8)}<1\right)$.

Reaction times were submitted to a within-participants ANOVA with TMS Time (pre, post), TMS Site (EBA, sham), and Trial Type (Hit, CR) as factors. There was a significant main effect of Time, $F_{(1,8)}=18.6, p<0.01, \eta_{p}{ }^{2}=0.70$, caused by speeding up of response times when TMS was applied during the preparatory interval $(457 \mathrm{~ms}(\mathrm{SE}=28)$ ) compared with the postinterval $(504 \mathrm{~ms}(\mathrm{SE}=27))$. No other main effects or interactions were significant (TMS Site $F_{(1,8)}=2.6, p=0.15, \eta_{p}{ }^{2}=0.24$, all other $F<1)$.

\section{Experiment 3}

Mean response time from accurate trials (93\% of trials) for which response time was within the range of 200-1000 ms, were submitted to an ANOVA with Task (car, people), TMS Site (EBA, TOS, sham), and Hemifield (left, right) as factors (Fig. 6). Significant main effects of Task, $F_{(1,15)}=4.9, p<0.05, \eta_{p}{ }^{2}=0.24$, and of TMS Site, $F_{(2,30)}=3.4, p<0.05, \eta_{p}{ }^{2}=0.18$, were qualified by an interaction of these two factors, $F_{(2,30)}=4.0, p<0.05, \eta_{p}{ }^{2}=$ 0.21 . No other main effects or interactions were significant, all $F<1.6$. The interaction of Task and TMS Site was followed up with separate one-way ANOVAs, collapsing across Hemifield, separately for each task. For the car task, there was no reliable effect of TMS Site, $F_{(2,30)}=1.6, p=0.23, \eta_{p}{ }^{2}=0.09$. In contrast, for the people task, there was a significant main effect of TMS Site, $F_{(2,30)}=4.4, p<0.05, \eta_{p}{ }^{2}=0.23$. In follow-up pairwise $t$ tests, the response times for EBA stimulation $(415 \mathrm{~ms}(\mathrm{SE}=18)$ were significantly slower than for TOS stimulation (386 ms ( $\mathrm{SE}=$ $11), t_{(15)}=2.2, p<0.05$. Response times for EBA stimulation
People Task

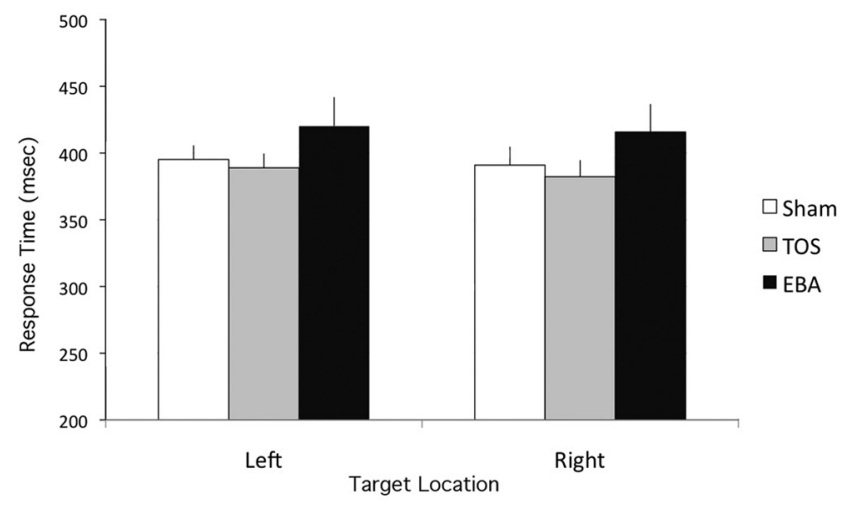

Car Task

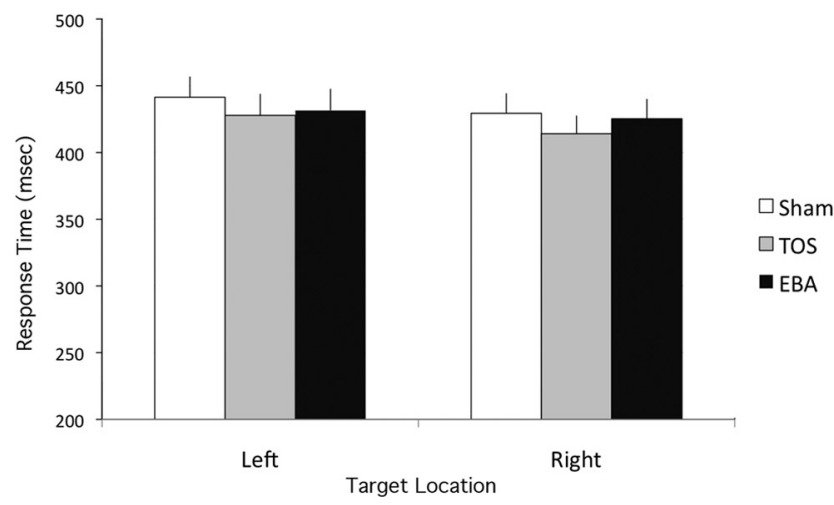

Figure 6. Results of Experiment 3. EBA stimulation, relative to TOS stimulation, increases response times to locate (left/right hemifield) a scene containing people, equivalently for targets in either hemifield. TMS had no significant effect on a control task requiring left/right localization of scenes containing cars.

were marginally significantly slower than for sham stimulation (394 $\mathrm{ms}(\mathrm{SE}=11), t_{(15)}=1.9, p=0.08$.

The mean error rates were submitted to a similar ANOVA. There was a significant main effect of TMS Site, $F_{(2,30)}=4.8, p<$ $0.05, \eta_{p}{ }^{2}=0.24$, and a significant interaction between Task $\times$ TMS Site, $F_{(2,30)}=3.7, p<0.05, \eta_{p}{ }^{2}=0.20$. Separate ANOVAs were performed for each task. For the car task, there were no significant effects, but the main effect of TMS Site was significant for the people task, $F_{(2,30)}=6.5, p<0.005, \eta_{p}{ }^{2}=0.30$. Error rates on this task were slightly but reliably $(p<0.05)$ higher during TMS over TOS $(8.1 \%(\mathrm{SE}=0.7 \%))$ compared with both TMS over EBA $(6.2 \%(\mathrm{SE}=0.7 \%))$ and sham $(5.6 \%(\mathrm{SE}=$ $0.6 \%)$ ). This effect of TOS stimulation, while not germane to the present study, is notable given the results of a recent TMS study on this region. Ganaden et al. (2013) found that while left TOS stimulation did not differentially interfere with a man-made/natural scene categorization task, it did result in slower reaction times and lower accuracy rates, relative to a control condition. Further, Dilks et al. (2013) found that TMS over this region selectively impaired discrimination or categorization of highly similar or degraded buildings and scenes (relative to similar tasks on faces or objects). Person detection/discrimination was not tested.

\section{Discussion}

Human observers can efficiently detect abstractly specified targets in complex natural scenes, even when these are presented very briefly (Intraub, 1981; Thorpe et al., 1996; Li et al., 2002). 
Here we have demonstrated a causal role for a category-selective region in detecting its preferred stimulus class in complex scenes. TMS over rEBA reduced the sensitivity of observers (as measured by $\mathrm{D}$-prime) to detect people in briefly presented scene pictures. This reduced sensitivity was specific to EBA (compared with sham and/or TOS stimulation) and to person targets (compared with car targets). We found evidence that EBA stimulation is more effective immediately after stimulus presentation compared with immediately before presentation. TMS also affected decision criteria in Experiments 1 and 2, making participants slightly more conservative in Experiment 1, but slightly more liberal in Experiment 2. In both cases D-prime was reduced; these findings highlight the value of the signal-detection approach in separating contributions of sensitivity and response criterion to detection performance. Finally, we found evidence from a response-time measure that the effects of right hemisphere EBA stimulation extend to person detection in both hemifields. Collectively, these findings have implications for our understanding of the neural basis of natural scene categorization, and for understanding the properties of category-selective brain regions identified with fMRI.

Previous neuroimaging studies have shown that the detection of objects in natural scenes is associated with the evocation of distributed category-specific patterns of neural activity in the occipitotemporal cortex (Peelen et al., 2009; Peelen and Kastner, 2011). Interestingly, person selectivity in EBA was strongly modulated by task relevance: EBA was person selective when participants sought to detect people, but not when they detected the presence of cars in the same scenes, suggesting a role for EBA in person detection (Downing and Peelen, 2011). The present TMS results provide evidence for a causal involvement of the rEBA in the detection of people in natural scenes.

Our results also showed that TMS was more effective at impairing person detection when delivered immediately after $(+100$ and $+200 \mathrm{~ms})$ than immediately before $(-200$ and -100 $\mathrm{ms}$ ) the presentation of the target scenes. We speculate that the contribution of EBA to the present task is to on-line processing of the stimulus, rather than to anticipatory prestimulus activity. This is in line with the findings of a recent fMRI study, in which overall preparatory activity in EBA did not differ for trials on which participants prepared to detect people as compared with cars (Peelen and Kastner, 2011). Instead, it was shown that distributed activity patterns in object-selective cortex discriminated the two preparatory states, and that such preparatory activity patterns were correlated with object detection performance (Peelen and Kastner, 2011). Together with the present findings, this suggests that the preparation to detect people is reflected in distributed patterns partly outside EBA, with EBA subsequently being critical for deciding whether a scene picture contains a person or not.

Previous TMS studies on natural scene categorization have shown a causal role for early visual cortex in detecting animals in scenes (Camprodon et al., 2010; Koivisto et al., 2011). V1/V2 was causally involved in animal detection at two time intervals (100 and $220 \mathrm{~ms}$; Camprodon et al., 2010), both before and after lateral occipital cortex (LO) was implicated in the task (Koivisto et al., 2011). These results were interpreted as reflecting recurrent interactive processing, with both feedforward and feedback connections between LO and V1/V2 contributing to animal detection. Future studies could test whether recurrent processing is similarly important for the detection of people in scenes, and if so, whether EBA (rather than LO) is the source of feedback to early visual cortex during person detection.
Another TMS study on natural scene perception found that TMS over LO facilitated scene processing (Mullin and Steeves, 2011). In that study, rather than detecting the presence of object categories in scenes, participants indicated whether the scenes were natural or man-made environments. It is likely that this type of scene discrimination is performed based on global scene properties such as the global layout of the scene, the spatial frequency distribution of the image, or the overall amount of clutter (Oliva and Torralba, 2007). Processing such global features may rely on a different neuroanatomical pathway than that involved in detecting within-scene objects (Wolfe et al., 2011). Inhibitory connections between the object and scene pathways could then account for the facilitatory effects observed in Mullin and Steeves (2011). The dual-pathway hypothesis is supported by fMRI studies showing that activity patterns in lateral occipitotemporal cortex contain information about within-scene objects, while patterns in scene-selective areas such as parahippocampal place area, TOS, and retrosplenial cortex contain information about global scene layout (Goh et al., 2004; Walther et al., 2011; Kravitz et al., 2011; MacEvoy and Epstein, 2011; Park et al., 2011; Harel et al., 2013).

All previous TMS studies of EBA, as well as those of faceselective occipital and temporal regions, have tested single target stimuli that were presented in isolation against a simple, neutral background (Urgesi et al., 2004, 2007a,b; Pitcher et al., 2007, 2008, 2009; Candidi et al., 2008; Calvo-Merino et al., 2010). While these studies have uniformly shown selective disruption by TMS on tasks involving the preferred category of a region (as measured with fMRI and event-related potential, ERP), the use of such stimuli limits conclusions about a region's role in real-world vision.

Furthermore, in all previous TMS studies of the behavioral effects of EBA stimulation, participants performed a body discrimination task. Typically, a body (or body part) stimulus was presented, followed by a brief delay, followed by either one test image (task: same or different?) or two test images (task: which matches the sample?). TMS was delivered in the interval or during the test stimulus. A similar task protocol was also used in a study of neurological patients with occipitotemporal damage (Moro et al., 2008). Thus these findings, while important demonstrations of selectivity, leave open the question of whether EBA selectively contributes to the detection of people. The target stimuli (people) in the images tested here, when present, appeared in complex background scenes. They varied in number, position, posture, and identity, and could be occluded to varying degrees. The finding that EBA stimulation interferes with such a task argues strongly for a causal role in detecting other people, and further that this region contributes to a representation that captures general abstract visual features of the human body. Of course, these findings do not contradict an additional role for EBA in the discrimination of body shapes, or in short-term memory maintenance of body-related perceptual information.

Studies of body perception using ERP or intracranial electrophysiological recordings demonstrate a body-selective negative deflection in the region of EBA, peaking at about $190 \mathrm{~ms}$ after stimulus onset (Stekelenburg and de Gelder, 2004; Pourtois et al., 2007; Taylor et al. 2010, Thierry et al., 2006). Recent evidence shows that TMS over EBA modulates this N1 response, selectively increasing its amplitude only to images of bodies (Sadeh et al., 2011). Consistent with these ERP findings, our results showed that TMS was effective at impairing person detection when delivered at 100 and $200 \mathrm{~ms}$ after the presentation of the target scenes. 
Our final experiment showed that TMS to EBA delayed correct responses in a localization task. These results suggest that right hemisphere EBA is implicated in person detection across both sides of the visual field. A previous study using fMRI with multivariate pattern analysis (Chan et al., 2010) reported contralateral biases in the discrimination of body parts, with, for example, stronger discrimination via rEBA patterns of types of body parts presented in the left hemifield. Although superficially contradictory, the two sets of results are difficult to compare directly as they involve different measures on different tasks, operating over different timescales. Nonetheless these findings suggest further studies in which the laterality of TMS (left vs right EBA) is manipulated systematically. Such studies would shed light on the organization of spatial information in occipitotemporal regions, a topic of great recent interest (Niemeier et al., 2005; Hemond et al., 2007; MacEvoy and Epstein, 2007; Schwarzlose et al., 2008; Kravitz et al., 2010; Taylor and Downing, 2011). They could also inform us about differences between left and right EBA in the response to body perspectives (Chan et al., 2004; Saxe et al., 2006) and haptic perception (Costantini et al., 2011). Finally, given recent reports that the lateralization of EBA responses depends on gender and handedness (Aleong and Paus, 2010; Willems et al., 2010) these factors should also be examined systematically.

In summary, activity in the rEBA area contributes causally to the detection of people in natural scenes. This is consistent with our previous proposal (Downing and Peelen, 2011) that this region, together with the fusiform body area (Peelen and Downing, 2005), creates a mid-level representation of the shape and posture of perceived human form. In doing so, it extracts vital information rapidly and efficiently, performing a core function of the social brain.

\section{References}

Aleong R, Paus T (2010) Neural correlates of human body perception. J Cogn Neurosci 22:482-495. CrossRef Medline

Bindemann M, Scheepers C, Ferguson HJ, Burton AM (2010) Face, body, and center of gravity mediate person detection in natural scenes. J Exp Psychol Hum Percept Perform 36:1477-1485. CrossRef Medline

Calvo-Merino B, Urgesi C, Orgs G, Aglioti SM, Haggard P (2010) Extrastriate body area underlies aesthetic evaluation of body stimuli. Exp Brain Res 204:447-456. CrossRef Medline

Camprodon JA, Zohary E, Brodbeck V, Pascual-Leone A (2010) Two phases of V1 activity for visual recognition of natural images. J Cogn Neurosci 22:1262-1269. CrossRef Medline

Candidi M, Urgesi C, Ionta S, Aglioti SM (2008) Virtual lesion of ventral premotor cortex impairs visual perception of biomechanically possible but not impossible actions. Soc Neurosci 3:388-400. CrossRef Medline

Chan AW, Peelen MV, Downing E (2004) The effect of viewpoint on body representation in extrastriate body area. Neuroreport 15:2406-2410. Medline

Chan AW, Kravitz DJ, Truong S, Arizpe J, Baker CI (2010) Cortical representations of bodies and faces are strongest in commonly experienced configurations. Nat Neurosci 13:417-418. CrossRef Medline

Costantini M, Urgesi C, Galati G, Romani GL, Aglioti SM (2011) Haptic perception and body representation in lateral and medial occipitotemporal cortices. Neuropsychologia 49:821-829. CrossRef Medline

Dilks DD, Julian JB, Paunov AM, Kanwisher N (2013) The occipital place area is causally and selectively involved in scene perception. J Neurosci 33:1331-1336a. CrossRef Medline

Downing PE, Peelen MV (2011) The role of occipitotemporal bodyselective regions in person perception. Cogn Neurosci 2:186-226. CrossRef

Downing PE, Jiang Y, Shuman M, Kanwisher N (2001) A cortical area selective for visual processing of the human body. Science 293:2470-2473. CrossRef Medline

Downing PE, Bray D, Rogers J, Childs C (2004) Bodies capture attention when nothing is expected. Cognition 93:B27-B38. CrossRef Medline
Epstein RA, Higgins JS, Thompson-Schill SL (2005) Learning places from views: variation in scene processing as a function of experience and navigational ability. J Cogn Neurosci 17:73-83. CrossRef Medline

Ganaden RE, Mullin CR, Steeves JKE (2013) Transcranial Magnetic Stimulation to the Transverse Occipital Sulcus Affects Scene but not Object Processing. J Cogn Neurosci. Advanced online publication. doi:10.1162/jocn_a_00372. CrossRef

Goh JO, Siong SC, Park D, Gutchess A, Hebrank A, Chee MW (2004) Cortical areas involved in object, background, and object-background processing revealed with functional magnetic resonance adaptation. J Neurosci 24:10223-10228. CrossRef Medline

Harel A, Kravitz DJ, Baker CI (2013) Deconstructing visual scenes in cortex: gradients of object and spatial layout information. Cereb Cortex 23:947957. CrossRef Medline

Hemond CC, Kanwisher NG, Op de Beeck HP (2007) A preference for contralateral stimuli in human object- and face-selective cortex. PLoS ONE 2:e574. CrossRef Medline

Hodzic A, Kaas A, Muckli L, Stirn A, Singer W (2009) Distinct cortical networks for the detection and identification of human body. Neuroimage 45:1264-1271. CrossRef Medline

Intraub H (1981) Rapid conceptual identification of sequentially presented pictures. J Exp Psychol 7:604-610.

Kanwisher N, Yovel G (2006) The fusiform face area: a cortical region specialized for the perception of faces. Philos Trans R Soc Lond B Biol Sci 361:2109-2128. CrossRef Medline

Keel JC, Smith MJ, Wassermann EM (2001) A safety screening questionnaire for transcranial magnetic stimulation. Clin Neurophysiol 112:720. CrossRef Medline

Koivisto M, Railo H, Salminen-Vaparanta N (2011) Transcranial magnetic stimulation of early visual cortex interferes with subjective visual awareness and objective forced-choice performance. Conscious Cogn 20:288298. CrossRef Medline

Kontaris I, Wiggett AJ, Downing PE (2009) Dissociation of extrastriate body and biological-motion selective areas by manipulation of visualmotor congruency. Neuropsychologia 47:3118-3124. CrossRef Medline

Kravitz DJ, Kriegeskorte N, Baker CI (2010) High-level visual object representations are constrained by position. Cereb Cortex 20:2916-2925. CrossRef Medline

Kravitz DJ, Peng CS, Baker CI (2011) Real-world scene representations in high-level visual cortex: it's the spaces more than the places. J Neurosci 31:7322-7333. Medline

Li FF, VanRullen R, Koch C, Perona P (2002) Rapid natural scene categorization in the near absence of attention. Proc Natl Acad Sci U S A 99:95969601. Medline

MacEvoy SP, Epstein RA (2007) Position selectivity in scene- and objectresponsive occipitotemporal regions. J Neurophysiol 98:2089-2098. CrossRef Medline

MacEvoy SP, Epstein RA (2011) Constructing scenes from objects in human occipitotemporal cortex. Nat Neurosci 14:1323-1329. CrossRef Medline

Moro V, Urgesi C, Pernigo S, Lanteri P, Pazzaglia M, Aglioti SM (2008) The neural basis of body form and body action agnosia. Neuron 60:235-246. CrossRef Medline

Mullin CR, Steeves JK (2011) TMS to the lateral occipital cortex disrupts object processing but facilitates scene processing. J Cogn Neurosci 23: 4174-4184. CrossRef Medline

Niemeier M, Goltz HC, Kuchinad A, Tweed DB, Vilis T (2005) A contralateral preference in the lateral occipital area: sensory and attentional mechanisms. Cereb Cortex 15:325-331. Medline

Oliva A, Torralba A (2007) The role of context in object recognition. Trends Cogn Sci 11:520-527. CrossRef Medline

Park S, Brady TF, Greene MR, Oliva A (2011) Disentangling scene content from spatial boundary: complementary roles for the parahippocampal place area and lateral occipital complex in representing real-world scenes. J Neurosci 31:1333-1340. CrossRef Medline

Peelen MV, Downing PE (2005) Selectivity for the human body in the fusiform gyrus. J Neurophysiol 93:603-608. Medline

Peelen MV, Downing PE (2007) The neural basis of visual body perception. Nat Rev Neurosci 8:636-648. CrossRef Medline

Peelen MV, Kastner S (2011) A neural basis for real-world visual search in human occipitotemporal cortex. Proc Natl Acad Sci US A 108: 12125-12130. CrossRef Medline 
Peelen MV, Fei-Fei L, Kastner S (2009) Neural mechanisms of rapid natural scene categorization in human visual cortex. Nature 460:94-97. CrossRef Medline

Pitcher D, Walsh V, Yovel G, Duchaine B (2007) TMS evidence for the involvement of the right occipital face area in early face processing. Curr Biol 17:1568-1573. CrossRef Medline

Pitcher D, Garrido L, Walsh V, Duchaine BC (2008) Transcranial magnetic stimulation disrupts the perception and embodiment of facial expressions. J Neurosci 28:8929-8933. CrossRef Medline

Pitcher D, Charles L, Devlin JT, Walsh V, Duchaine B (2009) Triple dissociation of faces, bodies, and objects in extrastriate cortex. Curr Biol 19: 319-324. CrossRef Medline

Pourtois G, Peelen MV, Spinelli L, Seeck M, Vuilleumier P (2007) Direct intracranial recording of body-selective responses in human extrastriate visual cortex. Neuropsychologia 45:2621-2625. CrossRef Medline

Ro T, Friggel A, Lavie N (2007) Attentional biases for faces and body parts. Visual Cogn 15:322-348. CrossRef

Russell BC, Torralba A, Murphy KP, Freeman WT (2008) LabelMe: a database and web-based tool for image annotation. Int J Comput Vis 77:157173. CrossRef

Sadeh B, Pitcher D, Brandman T, Eisen A, Thaler A, Yovel G (2011) Stimulation of category-selective brain areas modulates ERP to their preferred categories. Curr Biol 21:1894-1899. CrossRef Medline

Saxe R, Jamal N, Powell L (2006) My body or yours? The effect of visual perspective on cortical body representations. Cereb Cortex 16:178-182. CrossRef Medline

Schwarzlose RF, Swisher JD, Dang S, Kanwisher N (2008) The distribution of category and location information across object-selective regions in human visual cortex. Proc Natl Acad Sci U S A 105:4447-4452. CrossRef Medline

Stein T, Sterzer P, Peelen MV (2012) Privileged detection of conspecifics: evidence from inversion effects during continuous flash suppression. Cognition 125:64-79. CrossRef Medline

Stekelenburg JJ, de Gelder B (2004) The neural correlates of perceiving human bodies: an ERP study on the body-inversion effect. Neuroreport 15:777-780. CrossRef Medline

Talairach J, Tournoux P (1988) Co-planar stereotaxic atlas of the human brain: 3-dimensional proportional system - an approach to cerebral imaging. New York: Thieme Medical .

Taylor JC, Downing PE (2011) Division of labor between lateral and ventral extrastriate representations of faces, bodies, and objects. J Cogn Neurosci 23:4122-4137. CrossRef Medline

Taylor JC, Roberts MV, Downing PE, Thierry G (2010) Functional characterisation of the extrastriate body area based on the N1 ERP component. Brain Cogn 73:153-159. CrossRef Medline

Thierry G, Pegna AJ, Dodds C, Roberts M, Basan S, Downing P (2006) An event-related potential component sensitive to images of the human body. Neuroimage 32:871-879. CrossRef Medline

Thorpe S, Fize D, Marlot C (1996) Speed of processing in the human visual system. Nature 381:520-522. CrossRef Medline

Tsao DY, Livingstone MS (2008) Mechanisms of face perception. Annu Rev Neurosci 31:411-437. CrossRef Medline

Urgesi C, Berlucchi G, Aglioti SM (2004) Magnetic stimulation of extrastriate body area impairs visual processing of nonfacial body parts. Curr Biol 14:2130-2134. CrossRef Medline

Urgesi C, Calvo-Merino B, Haggard P, Aglioti SM (2007a) Transcranial magnetic stimulation reveals two cortical pathways for visual body processing. J Neurosci 27:8023-8030. CrossRef Medline

Urgesi C, Candidi M, Ionta S, Aglioti SM (2007b) Representation of body identity and body actions in extrastriate body area and ventral premotor cortex. Nat Neurosci 10:30-31. CrossRef Medline

Walther DB, Caddigan E, Fei-Fei L, Beck DM (2009) Natural scene categories revealed in distributed patterns of activity in the human brain. J Neurosci 29:10573-10581. CrossRef Medline

Walther DB, Chai B, Caddigan E, Beck DM, Fei-Fei L (2011) Simple line drawings suffice for functional MRI decoding of natural scene catergories. Proc Natl Acad Sci U S A 108:9661-9666. CrossRef Medline

Willems RM, Peelen MV, Hagoort P (2010) Cerebral lateralization of faceselective and body-selective visual areas depends on handedness. Cereb Cortex 20:1719-1725. CrossRef Medline

Wolfe JM, Võ ML, Evans KK, Greene MR (2011) Visual search in scenes involves selective and nonselective pathways. Trends Cogn Sci 15:77-84. CrossRef Medline 